\title{
ERRATUM \\ Opérations sur les cartes et métamorphoses de la catégorie des G-ensembles
}

C. LEGER

REVISTA MATEMATICA de la Universidad Complutense de Madrid, Volumen 4, número 1, 1991

\section{Page 48}

Septième paragraphe, dernière ligne: supprimer $\mathrm{Tg}$ :

Après le septième paragraphe, insérer le nowveau paragraphe:

Pour tout élément $g$ de $G$ on note $T g$ la translation à droite $x \rightarrow x . g$ de $G$.

Démonstration du lemme 6, troisième ligne:

remplacer $\quad y=g \cdot x \quad$ par $\quad y=g^{-1} \cdot x$

Démonstration du lemme 6, cinquième ligne:

remplacer gauche par droite

Page 49

Deuxième ligne:

remplacer $\quad g^{-1} \cdot x$ par $g . x$ 